\title{
Importance of medication adherence and factors affecting it
}

\author{
Dwajani S. 1,", Prabhu MR², Ranjana G. ${ }^{3}$, Sahajananda $\mathbf{H}^{4}$ \\ ${ }^{\mathbf{1}}$ Assistant Professor/Senior Research Associate, Dept. of Pharmacology/Central Research Lab, ${ }^{2}$ Research Assistant, Central \\ Ressearch Lab, ${ }^{3}$ Independent Researcher, Bangalore ${ }^{4}$ Professor \& HOD/Coordinator, Dept. of Anaesthesia/Central Research Lab, \\ ${ }^{1,2,4}$ Rajarajeswari Medical College and Hospital, Bangalore, Karnataka, India
}

*Corresponding Author:

Email: dwajani@gmail.com

\begin{abstract}
Non-adherence leads to considerable morbidity, mortality and avoidable health care costs. adherence averages to only $50 \%$ in patients suffering from chronic medical illness. Often, adherence and compliance are used interchangeably. Several determinants have been reported to influence adherence, like nature and duration of therapy, characteristics of disease, side-effects of medication, treatment cost, characteristics of health service facilities, the relation between the physician and patient, patient characteristics, patient's perspective about the illness and therapy. The determinants of non-adherence according to World Health Organization (WHO) are categorized into five factors: socioeconomic, health system-related, therapy-related, condition-related and patient-related. Issues associated with medication non-adherence, which are diverse and complex have been extensively researched for decades and are documented well throughout the literature. In light of this, our study is focused specifically on providing an overview of factors affecting adherence and commonly used measures of medication adherence.
\end{abstract}

Keywords: Adherence, Compliance, Treatment, Factors.

\section{Introduction}

It has been estimated by WHO in 2003 that, in developed countries' adherence averages to only $50 \%$ in patients suffering from chronic medical illness and in developing countries the problem is much higher., ${ }^{1,2}$ Non-adherence leads to considerable morbidity, mortality and avoidable health care costs. ${ }^{3}$ The term adherence is defined as "the extent to which patients treatment related behaviors (taking medication, following a diet, modifying habits or attending clinics) correspond to health care professional's advice. Compliance is defined as the extent to which patients follow the instructions, prescriptions and prescriptions given by health professionals or the extent to which the patient's behavior or attitude exactly matches with the prescriber's recommendations. The word 'compliance' comes from the Latin word "compiler", which means to fill up and hence to complete an action, or process. ${ }^{4}$

Often, adherence and compliance are used interchangeably. ${ }^{5}$ Term concordance introduced recently is predominantly used in United Kingdom that comes from Latin word concorder which means to agree. It is an agreement reached after negotiation between a patient and a health care professional that respects the beliefs and wishes of the patient in determining whether, when and how medicines are to be taken. Although reciprocal, this is an alliance in which the health care professionals recognize the primacy of patient's decision about taking the recommended medications. ${ }^{6}$ The term adherence will be mainly be used in this paper. Reduction in treatment benefits is seen with low adherence. Several determinants have been reported to influence adherence, like nature and duration of therapy, characteristics of disease, side-effects of medication, treatment cost, characteristics of health service facilities, the relation between the physician and patient, patient characteristics (socioeconomic factors), patient's perspective about the illness and therapy. ${ }^{7}$

NICE guidelines refer to two types of nonadherence: 'intentional' and unintentional. Patients' deciding not to follow treatment recommendations which includes deliberately omitting prescriber's advice, skipping or altering a dose or ceasing to take medication due to experienced side-effects is referred to as intentional nonadherence. When the patient is prevented by specific barriers outside patient control, which include failing to comprehend or understand instructions for use, an inability to pay for treatment, or simply forgetting to take medication is referred to as unintentional nonadherence. ${ }^{8}$

The determinants of non-adherence according to World Health Organization (WHO) are categorized into five factors: socioeconomic (e.g. poor socioeconomic status, illiteracy , unemployment), health system-related (poor medication distribution, inadequate or nonexistent reimbursement or a lack of feedback on performance), therapy-related (complexity of medical regimens, duration of treatments or the immediacy of beneficial effects), conditionrelated(severity of symptoms, rates of progression or level of disability), and patient-related (knowledge and beliefs, motivations to manage or confidence). ${ }^{9}$

In the absence of a gold standard, multiple tools are utilized to assess adherence. It has been found that the medication diaries are only of limited use to evaluate the adherence. This is because the majorities of patients do not follow instructions or fill diaries immediately before their physician appointments. ${ }^{4}$ It has also been reported that the pills or refill count may overestimate 
adherence, since patients may discard pills, instead of taking medication. ${ }^{10}$ In comparison to the intake, the computerized pharmacy databases are more informative about medication acquisition. Patients' or caregiver's reports are subjective and may overestimate the adherence. It has been further reported that the medication intake under direct supervision can only be accomplished at once-daily regimens, in hospitalized patients or for patients under continuous nursing care. Adherence questionnaires are commonly used in clinical practice. ${ }^{11}$ Issues associated with medication non-adherence, which are diverse and complex have been extensively researched for decades and are documented well throughout the literature. In light of this, our study is focused specifically on providing an overview of factors affecting adherence and commonly used measures of medication adherence.

\section{Factors affecting Adherence}

According to the World Health Organization (WHO), non-adherence to the medication is one of the major clinical problem in management of patients with chronic illness. Adherence is a multidimensional phenomenon determined by the interaction of several different factors, termed "dimensions" by the WHO.

Table 1: Factors affecting adherence ${ }^{9}$

\begin{tabular}{|c|c|}
\hline Factors & Description \\
\hline Social/Economic & $\begin{array}{l}\text { 1. Better adherence observed in patients who have social support from family, } \\
\text { friends or caregiver who can assist with medication regimens. } \\
\text { 2. Lack of family or social support network unstable living environments like } \\
\text { homelessness, limited access to health care facilities, inability or difficulty in } \\
\text { accessing pharmacy, lack of financial resources, medication cost, cultural and } \\
\text { lay beliefs about illness, treatment and burdensome work schedules have all } \\
\text { been associated with reduced adherence rates. }\end{array}$ \\
\hline $\begin{array}{l}\text { Provider-patient } \\
\text { /Health care system }\end{array}$ & $\begin{array}{l}\text { 1. The best relationship of doctor-patient is one of the important health care } \\
\text { system related factor that has a more positive impact on medication } \\
\text { adherence. } \\
\text { 2. Poor or lack of proper communication regarding the beneficial effect of } \\
\text { taking medication, instructions for use and side effects of medications may } \\
\text { also lead to nonadherence, especially in elderly patients with memory } \\
\text { problems. }\end{array}$ \\
\hline Condition-r & $\begin{array}{l}\text { 1. Adherence to treatment regimens declines significantly over time in patients } \\
\text { suffering from chronic illness (high blood pressure, osteoporosis and } \\
\text { hyperlipidemia) which requires long term drugs administration. } \\
\text { 2. This decline is mainly because few or no symptoms. It is important that the } \\
\text { patient understands the illness and must know what will happen without } \\
\text { treatment. }\end{array}$ \\
\hline Therapy- & $\begin{array}{l}\text { 1. The complexity of the medication regimen which includes taking the number } \\
\text { of concurrent medications and number of daily doses required; duration of } \\
\text { therapy, lack of immediate benefit of therapy and treatment interferes with } \\
\text { lifestyle and side effects have been associated with decreased the rate of } \\
\text { adherence. }\end{array}$ \\
\hline Patient-related & $\begin{array}{l}\text { 1. Physical impairments such as visual, hearing and cognitive impairments and } \\
\text { swallowing problems may increase the risk for nonadherence in elderly } \\
\text { patients. } \\
\text { 2. Lack of knowledge about the disease and understanding reasons for which } \\
\text { medication is needed; lack of motivation, apprehension about possible } \\
\text { adverse side effects and substance abuse may be associated with poor } \\
\text { medication adherence. }\end{array}$ \\
\hline
\end{tabular}

\section{Measuring adherence}

There are several available methods for measuring medication adherence. Measurement falls into two common categories direct and indirect. The direct measurement includes $-\mathrm{a}$. drug monitoring $b$. detection of the drug or its metabolites in biological fluid and c. direct observation therapy. These approaches are one of the most accurate methods of adherence measurement but are expensive. Moreover, variations in metabolism can give a false impression of adherence. The indirect measurements include self reports, pill counts, rates of prescription refills, assessment of the patient's clinical response and measurement of physiologic markers as well as patient diaries.

\section{Direct Measurement}

1. Home finger prick sampling ${ }^{12,13}$ : There is growing interest in dried blood spot (DBS) 
sampling in TDM. The capillary blood is obtained from a finger prick with an automatic lancet. The patients will be able to do the self finger prick after adequate training. After disinfection, the patient pricks his/her finger with a lancet and the first drop of blood is discarded because it may contain more tissue fluid. The next drop is collected on a premarked circle on a filter paper. The DBS is allowed to dry at room temperature and then it is transported to the laboratory. In laboratory, the homogeneity of the blood spot is assessed and the analytes are measured with an analytical technique. It is easy, less invasive technique and most analytes are stable in dried blood spot. The disadvantages of this method are only small volumes of samples are available for analysis, the risk of contamination and lack of spare samples.

2. Segmental hair analysis of hair samples ${ }^{14}$ : New analytical, sensitive methods have been developed for determination of drugs and their metabolites by collection of hair samples. The analysis of hair samples could be one of the important tools for TDM that detects the xenobiotics compounds in forensic science, but it has not attributed much attention in the clinical field. It has potential usefulness of sampling noninvasive technique, wide detection and possibility to determine various analytes.

3. Biological markers ${ }^{15}$ : The concentration of drugs and its metabolites can be measured in biological samples like serum and or plasma, rarely in saliva, milk or fat using biological assays. These measures are intrusive, costly and poor availability of the assays. The results are influenced by several factors other than adherence such as drug or food interactions, physiological variability, dosing schedules and half-life of biological analytes

4. Directly observed therapy ${ }^{16}$ : The direct observed therapy is a best way of helping people for better treatment adherence, which means that the trained health care worker or clinicians provides the prescribed drugs and observe whether the patient can take every dose. It helps the patients to complete their therapy as early as possible without gap, thus reduces the risk of incomplete treatment

\section{Indirect Measures}

1. Self-report measures (using questionnaires) ${ }^{16}$ : It is the most common method for assessing medication adherence in research and clinical practice, but there are queries about its validity and precision. The Self-report medication adherence comprises of variation in their questionnaires, recall periods and response to the treatment. Selfreports estimate medication adherence behavior with high specificity but low sensitivity than other assessment methods. The measures of self reports may enhance the validated scales, assessment of the proper construct, estimation improvement, recall facilitation, reduce social desirability and employ technology delivery. Self-report medication adherence provides valuable information with their limitations.

2. MMAS-4 and 8 (Morisky's medication adherence scale) ${ }^{\mathbf{1 7}, 18}$ : Morisky's Medication Adherence Scale (MMAS-4) is validated and widely used in the assessment of various diseases and in patients with low literacy. MMAS-4 has poor psychometric properties when compared to MMAS-8. MMAS-4 had a sensitivity of $81 \%$ and specificity of $44 \%$, while MMAS- 8 scale showed $93 \%$ sensitivity and $53 \%$ specificity in the initial validation in hypertensive patient population. This result indicates that. MMAS- 8 considered as most accepted self-report measure for medication adherence. Therefore, it is recommended to serve as a screening tool for validated conditions in the clinic setting.

3. Medical outcome adherence study scale (MOA) $)^{19,20}$ : This scale was developed for certain disease conditions such as diabetes, hypertension and heart disease. The MOA scale is successfully used to measure the medication adherence in patients with heart failure and has been more reliable and validated. In this patients are asked questions with scores from 0 to 5 . The high score is directly related to higher medication adherence.

4. Brief medication Questionnaire (BMQ $)^{21}$ : The BMQ is exploring patient's medications, behavior and barriers to adherence of medication. It consists of three different screens, a 5-item Regime screen, Hill Bone Compliance scale, SEAMS (self efficacy for appropriate medication use scale), MARS (Medication adherence report scale), ARMS (Adherence to refills and medication scale) a 2item Belief screen and a 2-item Recall screen. This screening method used to assess how patients took each of their medications in the past, drug efficacy and remembering difficulties. Further reviewed this screening with its ability to allow self administration, evaluate multidrug regimens, while this questionnaire is more popular among healthcare professionals. It was first suggested for the disease condition such as diabetes and depression management. This scale seems to be more time-consuming compared to other questionnaires which makes difficult to be scored at the point of care.

a. Hill Bone Compliance scale ${ }^{22}$ : This scale could target the patients with antihypertensive medication only with their own limitation. The scale consists of 3 subscales including medication-taking behavior, able to keep the appointment, sodium intake and four-point likert-type scale. The Hill-Bone compliance scale has a higher performance for black than 
nonblack populations despite their high cultural sensitivity. This scale has been suggested and suitable for hypertension in the black population.

b. SEAMS (self efficacy for appropriate medication use scale) ${ }^{23}$ : Self-efficacy is defined as "belief or trust that one can successfully undertake a specific action, in order to achieve the desired result . A SEAM was developed by to evaluate the self efficacy of medication adherence in patients with lower literacy levels. It is a 13 question Likert-type of scale with a focus on chronic disease management. The reliability of this scale is measured by its internal consistency with coefficient alpha reliability at 0.89 and 0.88 on low and high literacy populations respectively. Therefore considered as an excellent selfreport tool for measuring adherence in chronic disease management.

c. MARS (Medication adherence report scale $)^{17}$ : MARS can be used to assess both beliefs and barriers to medication adherence. This scale is developed based on Drug Attitude Inventory (DAI), a common psychiatric adherence survey. The questionnaire has been incorporated from MAQ and reduces the deficiencies of DAI resulting ability to examine medication taking behaviors and attitudes toward medication with higher validity and reliability values. It comprises of 10 questions with simple scoring to evaluate patient's adherence behavior, attitude towards medication and disease control during the past week. The internal consistency reliability of MARS is unknown but still this scale has shown strong positive correlations compared to DAI and MAQ. This scale was first validated in patients with schizophrenia hence this scale is limited to use in patients with chronic mental illness.

d. Adherence to refills and medication scale (ARMS) ${ }^{17,25}$ : ARMS was developed, pilot tested, and administered to 435 patients in an inner-city primary care clinic with coronary heart disease. It's a 12 item scale which is valid and reliable when used in patients with chronic disease. It showed good performance characteristics even among patients with low literacy.

5. Basel Assessment of Adherence Scale for Immunosuppressants (BAASIS) ${ }^{26,27}$ : The BAASIS scale instrument was developed for assessment of adherence to immunosuppressive medication in adult transplant patients. This scale measures medication intake, skipping, timing $(>2$ hrs from prescribed time) and dose reduction of drugs. The recall period is limited to four weeks.
The BAASIS instrument scale consists of 4 questions with a 6-point scale for responses ranging from never (0) to every day (5). The BAASIS was developed to use in interviews, but it is also there in writen questionnaire version. The medication taking in the BAASIS has been validated for medication adherence to anti retroviral in patients with HIV.

6. Brief Adherence Rating Scale (BARS) ${ }^{28}$ : Is a clinician-administered adherence assessment tool It includes four items: three questions and an overall visual analog scale (VAS) to evaluate the percentage of doses taken by the patient in the past month (0\% to $100 \%)$. The VAS rating acts as a final adherence determination. Question 1 ask about patients' knowledge of their own medication regimen and episodes of missed medication taking, and include: number of prescribed doses per day ; question 2- number of days, over the past month, the patient did not take the prescribed doses and question 3- number of days, over the past month, the patient took less than the prescribed doses. A higher score on the BARS corresponds to better adherence.

7. Motivational interviewing ${ }^{29-31}$ : Motivational interviewing $[\mathrm{M}]$ is a communication style that evokes enthusiasm for betterment of patient's life. It is defines as "patients centered, directive counseling style, collaborative that helps in strengthening patients motive for change by evaluating and resolving ambivalence or resistance to change. MI consists of 3 components: collaboration, evocation and autonomy called "Spirit" of MI. on using MI as foundation, interaction becomes more collaborative, patients centered, direct and non judgemental.

8. Electronic Adherence monitoring (MEMS) ${ }^{32}$ : The Medication Event Electronic Monitoring System (MEMS) allows to assess the number of missed pill and adherence to a dosing schedule during a period of medication. The system monitors electronically when pill bottle is opened and researcher can periodically download the information to a computer. The availability and cost of this system limit the feasibility of its use.

9. POEMS (Polymedication electronic monitoring system) ${ }^{33}$ : The electronic medication event monitoring systems (MEMS) are currently limited to monitoring and assessment of single drug therapy. The new technology contains printed electronics self adhesive polymer film with loops of conductive wires that can be fixed to blister packaging. The smart components measure electrical resistance and records time of its change and data is transferred via a wireless device to a web based database. This technology was first developed to fit commercially available standard blister packs to avoid transfer of pills into an 
Electronic Compliances Measuring Device (ECMD). Later, developed electronic film technology to fit the rear side of a disposable multidose punch card. This packing consists of sealed calendar compartments with several medications to be taken together and thus avoiding patients to use multiple medication bottles. Currently, multidose punch cards are manually filled by pharmacists in Switzerland, Canada, United Kingdom, Germany, France and Australia.

10. Medminder ${ }^{34}$ : MedMinder Systems is a Newton, Mass based company founded in 2007 with the reason of giving services which simplifies medication management and improve medication adherence. "Maya" is an affordable and easy-to-use wireless pill dispenser which helps patients to take medications on time and provides reports to family and providers.

The new technology Ingestible Sensor System (ISS) allows direct and accurate measurement of medication adherence and captures medication intake dynamics through the ingestion of microsensors that can be incorporated into oral dose forms of active pharmaceuticals. It has been approved for use in the European Union (CE-mark) and in the United States of America. It is composed of ingestible event marker (IEM), a microsensor that becomes activated after ingestion, and adhesive personal monitor (APM) affixed to a torso that detects IEM once activated (A wireless Bluetooth-based interface within APM allows to transmit stored data to a smartphone, which in turn sends the data to a secured, centralized data storage and processing location via mobile telephony network.

11. Proteus raisin technology ${ }^{35}$ : Medication adherence applications was evaluated under three main smartphone operating systems Apple, Android, and Blackberry and the authors gave the highest ratings based on their wide range of features and enhanced levels of functionality for My MED Schedule, My MEDs, and RxmindMe. These apps can be easily implemented because they are inexpensive, scalable, accessible to anyone with smartphones, and do not require separate devices or packaging.

12. Smart ingestible sensor (pill) ${ }^{32}$ : Pill boxes and pill bottles come with sensors and are developed for monitoring the medication taking activity. It uses a 7 day multi compartment pill box embedding plungers in each compartment designed to detect the lid of boxes opening as plungers and activate a switch inside the pillbox that then triggers the micro controller. This system uses Bluetooth technology. However, the system donot ascertain if a pill is actually ingested or not by the patient.

13. Pill counts ${ }^{36}$ : Easiest method for calculating patient medication adherence is counting remaining number of pills and calculating the number of pills that the patient has taken. Data shows that this method may underestimate adherence in elderly populations and non-adherence is frequently difficult to assess with a simple count of pills on a certain date weeks to months after the prescription filled.

14. Database analysis ${ }^{37}$ : Databases are often used to evaluate medication usage. Secondary databases can be useful by offering the possibility for quick access to a set of individualized information from a large number of users. However, the clinical context is sometimes indispensable to compare and determine the information's validity

15. Continuous Multiple Interval Measure of Oversupply $(\mathbf{C M O S})^{38}$ : The CMOS is calculated concurrently with cumulative medication gap $(\mathrm{CMG})$. It is defined as the "observation period" and denotes an arbitrarily defined period, dates for the start and end of the data collection in which the target variables are examined. At the end of observation period, the accumulated gap is divided by total days between the start and end prescription to get CMG value for each patient. Similarly, the accumulated surplus is divided by the total days between the start and end of prescription to get the measure of surplus for each patient.

16. Medication Possession Ratio (MPR) ${ }^{39}$ : The MPR is measurement of medication adherence, defined as "ratio of the number of doses dispensed which is directly related to dispensing period and measures measure the percentage of time an individual has possession of medications". The MPR is calculated by the number of days of medication supplied within the dispensing (refill) interval / number of days in dispensing (refill) interval. It requires at least two dispensing refill dates for evaluation of MPR. The two forms of MPR that can be calculated such as fixed and variable MPR.

17. Variable MPR (VMPR $)^{40}$ : The VMPR is calculated by the number of days for prescribed medication was available between the first and last refill in the observation year divided by the number of elapsed days inclusive of last prescription.

18. Fixed MPR (FMPR) ${ }^{40}$ : The calculation is similar to VMPR, indicates number of days for which prescribed medication was available during the observation year.

19. Medication possession ratio modified ${ }^{41,42}:$ MPRm is used for determination of the medication supply. If the patients are having MPRm $>1.20$ are defined as that the patient receiving a medication oversupply. The MPRm is calculated by dividing the total number of days' supply of a specific generic name of medication for a patient divided by number of days from the first to last dispensation and plus the number of days supply in last dispensation. The MPRm is slightly modified 
from MPR by changing the formula from a prespecified time period (such as 1-year follow-up period) to the number of days from first to last dispensation, plus the number of days' supply in the last dispensation.

20. Continuous Measure of Medication Gaps (CMG) $^{\mathbf{4 3}}$ : CMG is a well validated method for evaluation of secondary adherence (adherence among ongoing users) for a periods bounded by medication dispensing; the proportion of time for which a patient received insufficient medication supplies (gap measure) ${ }^{[25]}$. The proportion of days for which a patient without sufficient medication supply across refill intervals beginning with the first and last dispensing before end of the follow up. CMG measures are calculated by dividing the total number of days in treatment gaps by the duration of the time period of interest in order to recognize any time intervals without drug exposure.

21. Proportion of days covered (PDC) ${ }^{43}$ : $P D C$ is a newer methodology of calculating adherence than the MPR but it has been extensively studied in recent years. The PDC calculation is based on the filling dates and supply days for each fill of a prescription hence it is differs from MPR. The proportion of days covered is in the measurement period covered by prescription claims for the same medication or another in its therapeutic category within a calendar range. The level of PDC has a reasonable likelihood of achieving most of the potential clinical benefit (i.e. $80 \%$ for diabetes and cardiovascular drugs; $90 \%$ for anti retrovirals drugs)

22. Continuous, Single Interval Measure of Medication Acquisition $(\mathbf{C S A})^{\mathbf{4 3}}$ : CSA is determined by the days' supply obtained in each interval over the total days in the interval ${ }^{[25]}$. Bias occurs when the patient gets more than one refill a day or when refill is close to the day of completion

23. Refill Compliance Rate $(\mathbf{R C R})^{\mathbf{3 8}}$ : The RCR can measure percentage of coverage. Calculated by fraction's numerator specifies the number of days on which the patient has medication available (days' supply) like MPRm, CSA etc. It is calculated and validated by sum of the amount during the observation period dividing by amount to take per day according to prescription and division of period between first and last dispensing multiplies by hundred.

24. Dates between Fills Adherence Rate $(\mathrm{DBR})^{38}$ : It is also measures the percentage of coverage like RCR by using a device for adjustment. It is calculated by period between first and last dispensing - days' supply divided by period between first and last dispensing multiplies by hundred.
25. Compliance Rate $(\mathbf{C R})^{38}$ : The $C R$ is used to measure the percentage of coverage (including excess) in a known and defined dispensing interval. It has been validated by days' supply in all the dispensing episodes except for last dividing by period between first and last dispensing in days multiples by hundred.

\section{Methods employed to improve adherence to medication}

The efficacy of medication and adherence to therapeutic routine determines the effectiveness of treatment. Several studies have shown that simple interventions can facilitate to improve adherence. ${ }^{44}$ Interventions promoting adherence has been classified under a mnemonic SIMPLE in a study done by Atreja et al. ${ }^{45}$

Simplifying the Regimen: Treatment regimen complexity can sometimes affect medication adherence. Several strategies can be used to simplify a regimen which has become a well standardized routine. This practice is more important for physicians to use simple, everyday language and have the patient repeat the instructions to ensure proper understanding of long term medication adherence. These regimens can be simplified for better, clearer understanding by end users or physician without altering the therapeutic intent of the regimen. ${ }^{45}$ Several adherence aids are available to help patients organize their medications (e.g. medication boxes) and remember dose time (alarms). Microelectronic devices can give feedback to the patients whether they are taking their medications as prescribed. In instances such as administering insulin injection, administering eye drops and pressurized inhalers and applying topical preparations, patients can also use devices designed to improve adherence etc. The advantages of simplification of regimen include reduced risk of treatment failure, long-term medication adherence and improved quality of life. ${ }^{46}$

Imparting Appropriate Knowledge: The research has constantly confirmed that understanding the patients' treatment conditions is directly associated to adherence, satisfaction, recall and type of information given to care takers by care givers. Several studies have shown that the patients do not always comprehend prescription instructions and frequently forget significant portions of what healthcare practitioners explained about treatment. ${ }^{46,47}$ By limiting instructions to three or four major points during each discussion. physicians can provide effective and valuable patient education. Particularly when explaining about diagnosis and giving instructions, simple everyday language may be used by the healthcare practitioner. The physician could supplement oral instructions with written materials. The patient's family members and their friends may involve in the discussion with physician about treatment pattern or diagnosis. This is especially true for millions of citizens with low literacy skills. ${ }^{48}$ 
Modifying Beliefs and Human Behaviour: In modern life style requires for giving worth to address patients' beliefs, intentions and self-efficacy (perceived ability to perform action). The physician can optimize behaviour change by ensuring the patients that perceive themselves to be at risk due to lack of adoption of healthy behaviour (perceived susceptibility), perceive their medical conditions to be serious (perceived severity), belief in the positive effect of the suggested treatment (perceived benefits) and have channels to address their fears and concerns (perceived barriers), and perceive themselves as having the requisite skills to perform the healthy behaviour (self-efficacy). ${ }^{45,49}$ Thus by knowing which of these belief presumed necessary for good adherence and the provider may suit the unique needs of each patient.

Patient Communication and trust: Patient communication involves in the interventions ranging from physician-patient communication, sending mail or telephonic reminder and to involving patients' families in the discussion with the clinicians. Of these, the more problematic is physician-patient communication..$^{50}$ At least $50 \%$ of care takers leave their doctors clinics because of not knowing what physicians exactly explained about the treatment. Studies have shown that the $50 \%$ of psycho social and psychiatric problems are missed by physicians due to lack of proper communication, $54 \%$ of patients' problems and $45 \%$ of patient concerns are neither neglected by the physician nor disclosed by the patient and $71 \%$ of patients stated that poor relationships for their malpractice claims. Studies devised the following suggestions after conducting a review of the physician-patient communications are ask a patient about his feelings and concerns and his view about psychological factors to the adherence and understanding the nature of the problem. ${ }^{45,51}$ Then provide valuable information about all areas that individual finds comfortable and encourage them to share self decision making attitude. At the same time, communication with the patient's family and friends, patient's own perception of social support are significantly related to adherence. The family's role becomes most important if patient is suffering from chronic disabling condition requiring continued support and understanding.

Leaving the Bias: A review studies have shown that there is no clear relationship between adherence and race, sex, educational experience, intelligence, marital status, occupational status, income and cultural background. ${ }^{52}$ Although some other studies have found a relation of adherence with sex and education but effect is small and may be overcome by training and education to the patient's level of understanding. Moreover, the fact that an individual's level of medication adherence may vary over time and between different aspects of treatment.

Evaluating Adherence: The evaluation of adherence is very important and hence it becomes imperative to measure and evaluate patient adherence reliably. It can be done by patient's self-reports which is the most widely used tool, pill counting and measuring serum or urine drug levels in some cases..$^{53}$ In general, patients can be very accurate in reporting whether they are adhering to their treatment regimens if physicians asked directly and regular assessment of patient adherence by itself leading to increased patient adherence..$^{54}$

\section{Conclusion}

Poor adherence to medication regimens is one of the most common problem, which contributes to substantial worsening of disease, death, with increase in the health care costs. Physicians should always identify the patients with poor adherence and help to enhance adherence by emphasizing the value of a patient's regimen by keeping the regimen simple, customizing the regimen to the patient's lifestyle. Enquiring the patients by non judgmentally about medication-taking behavior is a practical strategy for identifying poor adherence. A collaborative approach both y physician and patients is important to take care regarding the augments of adherence. Patients who undergo difficulty maintaining adequate adherence need more intensive strategies than do patients who have less difficulty with adherence, a more forgiving medication regimen, or both. Innovative methods of managing chronic diseases have had some success in improving adherence when a regimen has been difficult to follow. 99,125-127 New technologies such as reminders through cell phones and personal digital assistants and pillboxes with paging systems may be needed to help patients who have the most difficulty meeting the goals of a regimen.

\section{References}

1. E.Sabate, Adherence to Long-Term Therapies: Evidence for Action, World Health Organization, Geneva, Switzerland, 2003.

2. M.R. DiMatteo, Variations in patients adherence to medical recommendations: a quantitative review of 50 years of research. Medical Care. 2004;42(3):200-9.

3. McDonald H, Garg A, Haynes R. Interventions to enhance patient adherence to medication prescriptions. JAMA. 2002; 288(22):2868,

4. E. Lehane, G. McCarthy, "Medication non-adherenceexploring the conceptual mire," International Journal of Nursing Practice. 2009;15(1): 25-33.

5. Medicines Partnership. 2001. http://www.concordance.org (accessedon 27/05/2001)

6. Sohini Banerjee, Ravi Prasad Varma. Factors Affecting Non-Adherence among Patients Diagnosed with Unipolar Depression in a Psychiatric Department of a Tertiary Hospital in Kolkata, India. Depression Research and Treatment Volume. 2013;1-12.

7. National Institute for Health and Care Excellence (NICE), Medicines adherence: Involving patients in decisions about prescribed medicines and supporting adherence. NICE Clinical Guideline 76, January 2009.

8. WHO, "Adherence to long-term therapies: evidence for action,” Tech. Rep., World Health Organisation, Geneva, Switzerland, 2003. 
9. Dias, A. M., Cunha, M., Santos, A., Neves, A., Pinto, A., Silva, A, Castro, S. Adesão ao regime Terapêutico na Doença Crónica: Revisão da Literatura Millenium. 2011;40:201-9.

10. Yonan N, Matryszczuk R, Macheal A, Baynes A, Keevil BG, Monitoring of cyclosporine levels in transplant recepients using self administration finger prick sampling. Clin Transplant. 2006;20(2):221-5.

11. P.F.Hooper, Thomas Dresesen, Brian G Keevil, Sander Florman. Nat Clin Pract Nephr. 2009;5(3):E1.3

12. Alexander Muller, H.Jungen, Stefenac Iwerson, Martina Sterneck. H Anderson. Analysis of cyclosporine A in hair samples from liver transplanted patients. Ther Drug Monit.2013;0:1-9.

13. Aitio A, G.Becking, A Berlin, A Bernard, V Foa, D Kello, E Krug, A Leonard, G Nordberg. 1987. International workshop on indicators in human biological materials for assessing exposure to and/or biological effect of genotoxic chemicals. Copenhager. WHO regional office for Europe.

14. Kevin C. Farmer. Methods for measuring and monitoring medication regimen adherence in clinical trials and clinical practice. Clinical therapeutics.1999;21(6):107490.

15. Culing J, Marcel L. From Morisky to hill bone; self reports scales measuring adherence to medication. Coll antropol.2014;1:55-62.

16. D.E. Morisky, L.W Green, D M.Levine. Concurrent and predictive validity of a self reported measures of medication adherence. Medical care.1986;24(1):67-74.

17. Jia rong WV, Debra K Moser, Missok L Chung, Jerry A Lennie. Objectively measured but not self reported medication adherence independently predicts event free survival in patients with heart failure. J.Card fail.2008;14(3):203-10.

18. Dimalteo Mr, Sherbourne CD, Hay RD, Ordway L, Kravitz RL, Mc Glynn EA. Physicians characteristics influence patients adherence to medical treatment: Results from the medical outcome study. Health Psychol. 1993;12:93-102.

19. Bonnie L, Svarstada, Betty A Chewninga, Betsy L Sleath, Cecilia claessonc. The brief medication questionnaire : A tool for screening patients adherence and barrier to adherence. Patient education and counseling. 1999;37(2):113-24.

20. Kim MT, Hill MN, Bone LR, Levine DM. development and testing of the hill bone compliance to high blood pressure theraphy scale. Prog cardiovasc nurs.2000;15(3):90-6.

21. Bandura A. Social foundations of thought and action: A social cognitive theory. Eaglewood cliff. New Jersey: Prentice Hall; 1986.

22. Risser J, Jacobson TA, Kripalani S. Development and Psychometric Evaluation of the Self-Efficacy for Appropriate Medication Use Scale (SEAMS) in LowLiteracy Patients with Chronic Disease. J Nurs Meas. 2007;15(3):203-19.

23. Kripalani S, Risser J, Gatti Me, Jacobson TA. Development and evaluation of adherence to refills and medication scale ( ARMS) among low literacy patient with chronic disease.Value Health.2009;12(1):118-23.

24. Horne R, Frost S, Hankin M, Wright S, In the eye of beholder pharmacy students have more positive perception of medicines than students of other discipline. Int.J.Pharm.2001;9:85-9.

25. Glass. TR, DC Geest, Weber R, Vernazz Pl, Rickenbach, furrer $\mathrm{H}$. Et al., correlates of self reported non adherence to antiretroviral theraphy in HIV infected patients: the swiss HIV cohort study. J Acquir Immune deficiency syndrome. 2006;41(3):385-92.

26. Byerly MJ, Nakonezny PA, Rush AJ: The Brief Adherence Rating Scale (BARS) validated against electronic monitoring in assessing the antipsychotic medication adherence of outpatients with schizophrenia and schizoaffective disorder. Schizophr Res. 2008,100:60-9.

27. Treasure J. Motivational interviewing. Adv Psychiatr ther. 2004;10(5):331-71.

28. Lussier MT, Richard C. the motivational interview. Can fam physician.2007:53(12):2117-8.

29. Danielle M Miller, Tayla Rose, Jenny A. Behavioural interviewing:techniques to improve patients medication adherence. Consultant. 2016;56(8):702-5.

30. Murtadh Aldeer, Mehdi J. Richard p Martin. A review of medication adherence monitoring technologies. Applied system innovation. 2018;1(14):1-27.

31. Arnet, walter PN, Hersberger KE, Polymedication electronic monitoring system (POEMS) - a new technology for measuring adherence. Front in Pharmac. 2013;4(26):1-6.

32. Bethany. J. Foster, Ahna Pai, Huaqing Shao, susan Furth and for the TAKE -IT study group. The TAKE-IT study: aim, design and methods. BMC Nephrol.2014;15:139.

33. Wai Yin Lam, Paula Fresco. Medication adherence measures: An overview. Biomed res int.2015;1-12.

34. Osterberg, Blaschke T. Adherence to medication. New Eng J Med 2005;353(5):487-97.

35. Steiner J. F., Prochazka A. V. The assessment of refill compliance using pharmacy records: methods, validity, and applications. Journal of Clinical Epidemiology. 1997;50(1):105-116.

36. Hess L. M., Raebel M. A., Conner D. A., Malone D. C. Measurement of adherence in pharmacy administrative databases: a proposal for standard definitions and preferred measures. Annals of Pharmacotherapy. 2006;40(7-8):1280-1288. doi: 10.1345/aph.1h018.

37. Andrade S. E., Kahler K. H., Frech F., Chan K. A. Methods for evaluation of medication adherence and persistence using automated databases. Pharmacoepidemiology and Drug Safety. 2006;15(8):565-74. doi: 10.1002/pds.1230.

38. Chris M Kozma, Michael Dickson, Amy L Phillips, and Dennis M Meletiche. Medication possession ratio: implications of using fixed and variable observation periods in assessing adherence with disease-modifying drugs in patients with multiple sclerosis. Patient Prefer Adherence. 2013;7:509-16.

39. Raebel MA, Schmittdiel J, Konieczny JL, Karter AJ, Steiner JF. Standardizing terminology and definitions of medication adherence and persistence in research employing electronic databases. Med Care 2013; 8 Suppl 3:S11-21.

40. Elisangela da Costa Lima-Dellamora, Claudia Garcia Serpa Osorio-de-Castro, Livia Gonçalves dos Santos Lima Madruga and Thiago Botelho Azeredo. Use of pharmacy records to measure treatment adherence: a critical review of the literature. Cad. Saude Publica vol.33 no.3 Rio de Janeiro 2017 Epub Apr 20, 2017.

41. Van Dulmen S, Sluijs E, van Dijk L, et al and International Expert Forum on Patient Adherence. Furthering patient adherence: a position paper of the international expert forum on patient adherence based on an Internet forum discussion. BMC Health Serv Res. 2008;8:47. 
42. Atreja A, Bellam N, Levy S. Strategies to enhance patient adherence: Making it simple. Medacapt Gen Med. 2005:7(1):4

43. Ashish Atreja, Naresh Bellam, Susan R. Levy. Strategies to Enhance Patient Adherence: Making it Simple. Med Gen Med. 2005;7(1): 4.

44. Hall JA, Roter DL, Katz NR. Meta-analysis of correlates of provider behavior in medical encounters. Med Care. 1988;26:657-75.

45. Sarriff A, Aziz NA, Hassan Y, Ibrahim P, Darwis Y. A study of patients' self-interpretation of prescription instructions. J Clin Pharm Ther. 1992;17:125-8.

46. Katz JR. Back to basics: providing effective patient teaching. Am J Nurs. 1997;97:33-6.

47. Roter DL, Hall JA, Merisca R, Nordstrom B, Cretin D, Svarstad B. Effectiveness of interventions to improve patient compliance: a meta-analysis. Med Care. 1998;36:1138-61.

48. Frankel R, Beckman H. Evaluating the patient's primary problem(s) In: Stewart M, Roter D, editors.

Communicating With Medical Patients. Newbury Park, Calif: Sage Publications;1989. pp. 86-98.

49. Stewart MA, McWhinney IR, Buck CW. The doctor/patient relationship and its effect upon outcome. J R Coll Gen Pract. 1979;29:77-81.

50. Blake PG, Korbet SM, Blake R, et al. A multicenter study of noncompliance with continuous ambulatory peritoneal dialysis exchanges in US and Canadian patients. Am J Kidney Dis. 2000;35:506-14.

51. Duong M, Piroth L, Grappin M, et al. Evaluation of the Patient Medication Adherence Questionnaire as a tool for self-reported adherence assessment in HIV-infected patients on antiretroviral regimens. HIV Clin Trials. 2001;2:128-35. 